\title{
Cloud middleware and services-a systematic mapping review
}

\author{
Isaac Odun-Ayo ${ }^{1}$, Marion Adebiyi ${ }^{2}$, Olatunji Okesola ${ }^{3}$, Olufunke Vincent ${ }^{4}$ \\ ${ }^{1}$ Department of Computer and Information Sciences, Covenant University, Ota, Nigeria \\ ${ }^{2}$ Department of Computer Science, Landmark University, Omu-Aran, Nigeria \\ ${ }^{3}$ Department of Computational Sciences, First Technical University, Ibadan, Nigeria \\ ${ }^{4}$ Department of Computer Science, Federal University of Agriculture, Abeokuta, Nigeria
}

\begin{tabular}{|c|c|}
\hline Article Info & ABSTRACT \\
\hline Article history: & Cloud computing currently plays a crucial role in the delivery of vital \\
\hline Received Nov 12, 2020 & information technology services. A unique aspect of cloud computing is the \\
\hline Revised Mar 17, 2021 & cloud middleware and other related entities that support applications and \\
\hline Accepted Aug 23, 2021 & regards cloud middleware and services at all levels, and thus needs analysis \\
\hline Keywords: & $\begin{array}{l}\text { paper is to perform a systematic mapping for studies that capture cloud } \\
\text { computing middleware, stacks, tools and services. The methodology adopted }\end{array}$ \\
\hline Cloud computing & for this study is a systematic mapping review. The results showed that more \\
\hline Cloud delivery network & In the contribution facet were published with tool, model, method and \\
\hline Cloud middleware & $\begin{array}{l}\text { in terms of tool, evaluation and solution research had the largest number of } \\
\text { inten }\end{array}$ \\
\hline Cloud tools & articles with $14.17 \%$ and $26.77 \%$ respectively. A striking feature of the \\
\hline Services & systemic map is the high number of articles in solution research with respect \\
\hline Services at all layers & to all aspects of the features applied in the studies. This study showed clearly \\
\hline Systematic mapping & $\begin{array}{l}\text { that there are gaps in cloud computing middleware and delivery services that } \\
\text { would interest researchers and industry professionals desirous of research in } \\
\text { this area. }\end{array}$ \\
\hline
\end{tabular}

This is an open access article under the CC BY-SA license.

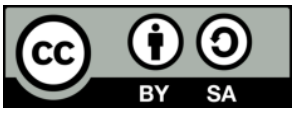

\section{Corresponding Author:}

Isaac Odun-Ayo

Department of Computer and Information Sciences

Covenant University

Ota, Nigeria

Email: isaac.odun-ayo@ covenantuniversity.edu.ng

\section{INTRODUCTION}

Cloud computing is characterized as a type of distributed and parallel system that consists of a series of dynamically supplied interlinked and virtualized computers which are provided by the service provider as several centralized computing resources with adequate service level agreements [1]. Cloud computing provides environments for managing resources concerning flexible infrastructure, middleware, application development frameworks and business applications. Cloud services are operated as pay-as-go or free vendor services. Software-as-a-service (SaaS), platform-as-a-service (PaaS), and infrastructure-as-a-service (IaaS) are the primary categories of cloud offerings. There are four cloud architecture models, in addition to the cloud services, which are private, public, community, and hybrid clouds which are used to supply a variety of services that enhances operations in data centres, internet of things, edge and fog computing [2].

The cloud middleware and services at all layers (XaaS) enhance operations on the cloud by connecting computers and devices to either cloud mobile or web applications which is also relevant to performance analysis of such aplications [3], [4]. In the traditional computing environment, the entire stack 
which consists of applications, data, runtime, middleware, operating system, virtualization, servers, storage and networking all reside in the data centre under the control of the organisation. Conversely, this entire stack is now supplied via a cloud service provider (CSP) under SaaS. The middleware is under the control of the user in PaaS, but managed by the CSP under IaaS. Various CSPs like Microsoft, Google, IBM and others have highly robust middleware and other microservices made available to user. Express is a server-side web framework for Node.js which helps to create APIs very quickly and easily by using hypertext transfer protocol. Express has built-in middleware functions, but middleware also comes from third-party packages and custom packages. For example, the IBM middleware functions in Express have access to the request and response objects.

CloudSim offers the ability to seamlessly model, simulate and experiment with evolving cloud computing infrastructure and application services, with a modern widespread and extensible simulation framework [5]. This includes support of large-scale cloud computing network modeling and simulation, and an integrated framework for data center modeling, service providers, planning, and allocation policies. The CloudSim toolkit is used to model, simulate, and execute cloud-enhancing applications. It enables extension and policy specification of every component of the software stack as a completely personalizable device. It helps users to handle the complexity of the specification, delivery and design of resources in physical environments [6]. Cloud bus toolkit is a layered, seven module architecture at the top of the stack, utilizing Cloud instances for everyday applications that are used for various purposes [7].

Cloud resources are acquired via third-party brokering services that promote access to the infrastructure. The virtual voyager, architect, network, native and mobile mover. The virtual voyager comprises the SaaS, PaaS and IaaS stacks. A virtual voyager leverages technology to access virtual computer resources in addition to increased versatility. It takes advantage of better response time, thereby reducing costs [8]. An application delivery network $(\mathrm{ADN})$ is an integrated infrastructure that includes communication tools, layer of service packets and network layer services for host applications [9]. However, the trend now is a software defined network (SDN). By proposing new design based on the separation of control and data planes, SDN enhances solution to protocol design problem and configuration errors on large-scale distributed systems.

Cloud middleware is an interesting field of research and the choice of a research subject can be a difficult process. A systematic mapping evaluation presents an overview of the number of publications within a field of interest and categorization them by using a scheme that allows a description of the degree of publications to be published in various fields of study [10]. Here, a systematic mapping study is built by examining issues relating to cloud middleware, and services. Through this process, the number of publications and work in this area will be defined. This study was carried out by establishing three facets for the scheme, they include the contribution, topic, and research facets. The contribution facet considered method and model amongst others, while the research facet dealt with the kind of research carried out. The topic facet was completed by extracting key areas of cloud middleware, stacks, tools and services on the cloud. In this paper, section 2 examines related work. The materials and method applied in the study are provided in section 3. The results and discussion on the outcome of the mapping process are presented in section 4. Section 5 shows the conclusion and recommendation for further research.

\section{RELATED WORK}

Santos, et al. [11] conducted research on systematic mapping to enhance the utilization of computer science conceptual diagrams, and the outcome of this study aims at identifying and analyzing current work utilizing backward snowballing as well as manual processes using five digital libraries. Through analyzing properly, the research focused on the teaching and learning process of concept maps and the key goals were achieved. Digital archives and repositories were used for research in the field of study.

Odun-Ayo et al. [2] is a systematic mapping research of edge computing and the internet of things with the cloud. A combination of topical, research and contribution facets were used to analyze publications on applications, concerning tool and model. They were closely followed by publications on architecture of edge computing. Opinion papers were the least published in the aspects of architecture. These were followed by publications on evaluation and solution research related to application. Most papers on ubiquitous networks were in the area of validation research. Furthermore, there was more work on optimization in the areas of philosophical, valuation and experience studies, with all following the procedures in [12]. SCOPUS, ScienceDirect, Compedex, ACM DL and IEEExplore were used for the work's search strings.

Petersen et al. [13] examines the manner in which researchers perform the systematic mapping process and how recommendations are to be revised based on lessons derived from the systematic maps and the procedures for systematic literature review. Certainly, the authors performed a comprehensive mapping study on systematic maps, taking into account relevant patterns in systematic reviews. They found that 
several recommendations have been used and merged in the vast number of studies carried out leading to a systematic mapping studies with more valuable conclusions.

The authors in Brocke et al. [14] presented six major aspects for the effective planning and communication of design of research projects that are science-based. They also emphasized the importance of the review of literature during scientific investigation and in the ever-increasing sphere of information system. The authors addressed the challenges of literary research and offered suggestions on how to resolve these challenges. They also offered practical instructions and checklists to help scientists plan and coordinate their literature searches.

The study in Odun-Ayo et al. [15] systematically documented cloud computing models and implementation patterns. Design, service deployment, implementation, configuration, data protection, security and usage models were considered by the categorization framework. A total of 131 fundamental examinations was utilized and the guide was drawn up based on the ideas of [13]. ScienceDirect, ACM digital library, Springer and IEEExplore provided results from different search strings of the exploration.

The work in Odun-Ayo et al. [16] is a systematic mapping analysis of networking for cloud and data centre. In the work, only 119 articles of the 131 articles consulted gave a concise contribution, which in this context was broken down into five major areas which are processes and metrics, devices, models and approaches. The result indicated that out of the 119 articles, contributions based on process and model had the highest percentage of $38.66 \%$ and $26.05 \%$. However, contributions based on methods, tools, and metrics were $19.33 \%, 10.08 \%$, and $5.88 \%$ respectively. SCOPUS, ScienceDirect, Compedex, ACM DL and IEEExplore were utilized in the work's search strings.

The paper in Mahamad et al. [17] designed a cloud-based people counter which could be used for the tracking and business marketing utilizing Raspberry Pi embedded systems, such that the received data is transmitted to the Thing Speak, IoT platform. Performance evaluation of the system was done in a real-life environment to ascertain its efficiency and capability to enhance business decisions. The basic stages of the work are; development of people counting algorithms, application of the developed algorithms into the hardware, and the integration of the acquired data onto Thing Speak.

The study in Reshma and Chetanaprakash [18] performed a study of developments in the automotive infotainment system with vehicular cloud network for enhancement and protection of in-car systems for smart and advanced entertainment operations. It is the vehicle ad-hoc network and support of the cloud system that relies on the transmission of this infotainment service. This work offers a summary of the research being done to exploit the infotainment system to get a genuine representation of input device power, weakness and open-end problems. The research contribution was summarized into the infotainment system over vehicular, schema-based approach, security-based approach, and traffic-based scheme.

The research in Kumar and Shantala [19] is a broad research overview on data integrity and deduplication against privacy in cloud storage because of the frailty of cloud data, which demands improved security. Consequently, this work discussed the express research commitment towards the Integrity of records, privacy, and deduplication of information. This also helps to highlight new open research issues and to explore the possible direction in which the existing challenges can be addressed. Unrealistic assumptions, non-applicability towards external intruders, unconsidered computational costs, according to the work outlined open issues in this field.

The study in Farahzadi et al. [20] performed a cloud of things middleware survey. To offer IoT services, the cloud is pivotal to giving intelligent conditions in houses, structures, and towns. There are, however, problems such as handling, aggregating, and preserving the big data involved. Cloud computing has enabled IoT as a web of things (WoT), providing almost limitless web services to expand broad-based IoT platforms. Middleware is capable of improving the heterogeneity of objects leading to efficient communication between interfaces, operating systems, and architectures. The research study analyzed CoT middleware technologies; identified the key characteristics of middleware for exploring various architectural styles and service areas. Also, to recognize middleware relevant to CoT-based stages and, to provide a rundown of current difficulties and issues in the improvement of CoT-based middleware.

The research in Endol et al. [21] systematically examined high cloud availability and research challenges. Before reaching cloud service that is compliant with service level agreements, many challenges must be overcome. The work covers checks, load balancing, and redundancies for the enhanced quality of cloud service while adding several research problems in this field without neglecting network and middleware solutions. To identify methods that cover high-availability clouds, it adapted the systematic analysis suggested by [22]. The work involved requirement for assessment, research and search queries, research resources, inclusion criteria and exclusion criteria, the procedure of information extraction identifying primary studies, assessing the quality of studies and extracting relevant information and presenting an overview of studies.

Bulletin of Electr Eng \& Inf, Vol. 10, No. 5, October 2021 : 2696 - 2706 
The study in Birje et al. [23] is a cloud study focused on cloud computing ideas, technology, challenges, and health. This paper investigates the model of cloud computing in the area of domain, principles, technology, instruments, and diverse challenges. A systematic literary review of 77 selected papers, released between 2000 and 2015, was performed to explain correctly the complexities of the cloud computing model. Hence security was identified as the greatest problem, the topic is discussed in depth separately. The concepts discussed are interoperability, resource planning, protection, data leakage, resource sharing, load balancing, multi-tenancy virtualization, and service level agreement (SLA).

The research in Chiregi and Navimipour [24] is the investigation of the cloud trust assessment and top-notch techniques utilized in cloud. Also, certain processes have been evaluated in terms of credibility, safety, efficiency, efficiency, protection, complexity, and scalability. There were 224 papers listed, that were screened to 28 based on the selection process. The survey would enable scholars, researchers, and experts to understand the developments in trust assessment processes in cloud environments through the presentation of up-to-date knowledge and problem issues. The strategy for selecting the article involves an automated, keyword-based search. The online repositories reviewed for this work are ScienceDirect, Google Scholar, Springerlink, IEEExplore, Elsevier, Wiley, Springer, and Emerald.

The work in Muhasin et al. [25] reviewed the issues of security and privacy affecting critical cloud data and identified solutions to tackle challenges such as user access management, sensitive data protection privacy, user identity anonymity, and data file protection. To explore the fundamental targets of the proposed system, a pilot study utilizing an organized survey was led by specialists working in cloud security. This also provides a multi-level structure to enhance the management of cloud-based information systems. It is a systematic analysis of literature carried out in compliance with Kitchenham's guidelines [13]. The systematic literature review starts with the review, investigation discovers, data gathering and information assessment. The paper made inquiries regarding the effects of critical data management in cloud computing. From the related work discussed so far, it is obvious that no work has been done in this area of study, hence our work is novel. This is a new system that is being proposed, hence it is novel. From the author's perspective, there are no similar papers in this field of study. However, there are several related works on systematic mapping studies which have been discussed in this paper which apply similar methodology in arriving at their results.

\section{MATERIALS AND METHOD}

This study focuses on developing a systematic map of cloud computing middleware, stacks, tools, delivery network and services at all layers. It was carried out in [11], [26], based on the formal guidelines for systematic mapping studies. A systematic mapping study is a repeatable method in which resources for research purposes are collected and interpreted. For a proper systematic mapping study as defined in Figure 1 there are several required steps. Research questions have to be defined, which focuses on the scope of review to be conducted. All articles in the specific study area under examination are also checked. The papers are screened after the search to decide which paper is acceptable for the research. By examining the abstracts in the respective papers, a scheme for classification is designed with keywording. The data extraction process led to the creation of a systematic map. The above steps were involved in creating a comprehensive map of middleware, stacks, software, supply networks and resources at all levels of the cloud.

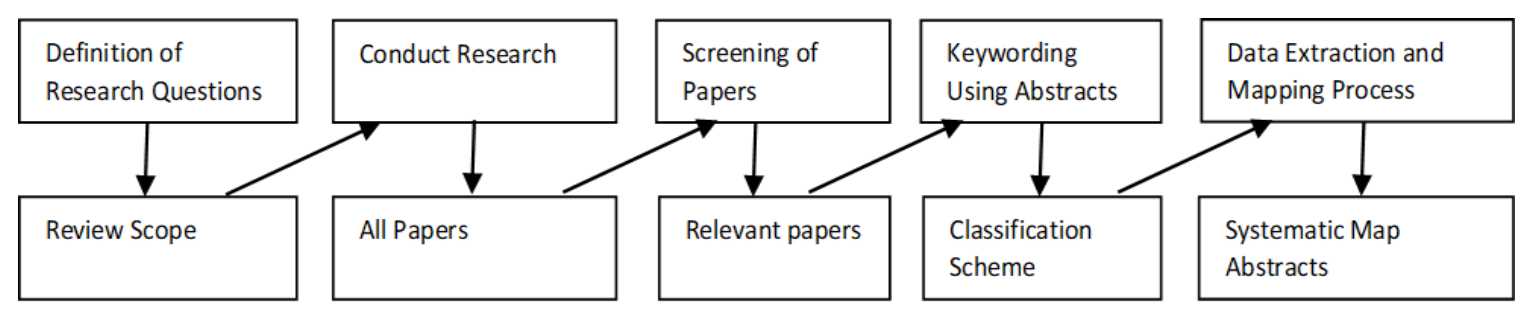

Figure 1. The systematic mapping process [13]

\subsection{Definition of research questions}

Systematic maps give a review of the amount and class of work that has been completed in a specific research domain. This allows the visualization of patterns over time by illustrating the rates of publications. The places in which the work was published in the study may also be important sometimes. It might likewise be fundamental sometimes to have a good knowledge of the areas in which publications have 
been done. These relevant issues decide the proper research question to be used in the study. The questions that govern the research are enumerated below:

- RQ 1: What aspect in cloud computing middleware, stacks, and services are examined as well as what number of papers encompass the different areas?

- RQ 2: What kinds of articles and in particular what is the unique pattern of papers published in this field?

\subsection{Conduction of primary studies research}

Primary studies are typically accomplished by exploration of major digital libraries. That is achieved by looking online for conference papers and articles. Searches were done on different digital advanced libraries accessible on-line to acquire papers for this systematic mapping study. The search did not concentrate on book and printed material. Due to the high impact of conference as well as journal publications of those databases, four databases were utilized for the hunt. The searched digital libraries are shown in Table 1.

Table 1. Electronic databases used for the systematic mapping study

\begin{tabular}{ll}
\hline Electronic databases & \multicolumn{1}{c}{ URL } \\
\hline ACM & http://dl.acm.org/ \\
IEEE & http://ieeexplore.ieee.org/xplore \\
SCIENCE DIRECT & http://www.sciencedirect.com/ \\
SPRINGER & http://www.springerlink.com/ \\
\hline
\end{tabular}

The search query was structured to elicit outcomes with respect to information, population and comparisons. All aspects of the study focused on the keywords used in the search string. The queries used on the leading digital libraries for this cloud-based middleware research, include stacks, tools, delivery networks and services in each layer:

(TITLE ("Cloud middleware") OR TITLE ("cloud computing middleware') or (ALL("cloud")AND ALL(middleware)))AND (TITLE (Stacks) OR TITLE (tools) OR TITLE ("'delivery networks") OR TITLE (XAAS) OR TITLE("'services at all layers"')).

For the search of the document metadata the above search string was used to ensure all relevant articles were obtained. All findings from the four digital repositories which relate to cloud computing and computer science were taken into account during the analysis. One hundred and twenty seven (127) papers were considered important to the inclusion from an initial list of 1,158 publications in the selected paper criteria, which were defined in the review emphasis and research question requirements. The research was carried out between 2001 and 2018.

\subsection{Inclusion and exclusion criteria paper screening}

Selection processes are used to ensure that the search and inclusion of all relevant research publications occurs. These processes were utilized to exclude irrelevant documents that pertains to the study, while papers pertaining to the study were included. This method also helps to exclude studies which have no direct answers to the research questions. Some abstracts related to the key issues without enough information and documents like these were also omitted. Furthermore, papers on tutorials, summaries, discussions, prefaces, editorials and presentation slides were excluded. The primary focal point of this study was considered important in inclusion of papers and also the secondary aspects were to a certain degree ex tracted too. Middleware, stack, platform, supply network and XaaS are the main focus of this report. Criteria for inclusion and exclusion therefore, are defined in Table 2.

Table 2. Inclusion and exclusion criteria

\begin{tabular}{|c|c|}
\hline Inclusion criteria & Exclusion criteria \\
\hline $\begin{array}{l}\text { In the abstract, middleware, stacks, software, distribution } \\
\text { networks and cloud services are addressed specifically. In } \\
\text { addition, the entire paper contributes to the study subject. }\end{array}$ & $\begin{array}{l}\text { This paper is beyond the field of cloud and computer science and } \\
\text { does not assist with middleware, stacks, software, supply networks } \\
\text { and infrastructure on all levels. The papers are not cloud-related. }\end{array}$ \\
\hline
\end{tabular}

\subsection{Keywording of abstracts}

The classification scheme used for the study is accomplished through a systematic process. Keywording was necessary in reducing the time required in developing the classification scheme for this work. Furthermore, keywording enables the researcher to consider all relevant publications through the 
classification scheme. The process involves the study of abstracts to identify concepts and keywords that relate to this study. After the extraction process keywords from numerous middleware-related articles were combined to provide comprehensive research insights. Afterwards, the categories for this study were established. However, in order for the analysis to be used with appropriate keywords it was often important to review the introduction, and conclusion of the publications. In conclusion, a keyword cluster was used for the evaluation of the various classes utilized in constructing the systematic map. Three aspects were utilized in this analysis of cloud middleware, stacks, networks and services. The first facets was based on the subject which was directly linked to the various aspects of the research. In the second facet, the types of contributions in the research paper were discussed in terms of the metric, model, instrument, system and procedure. The third facet comprised the research types undertaken.

\subsection{Categories and descriptions of facets on research types}

The third dimension dealt with the kind of work undertaken. A current classification of research approaches was explored [27]. The method includes the following definitions and categories.

a. Validation of research: the techniques used in the papers reviewed are unique but not yet implemented for example conducting an experiment in the laboratory.

b. Evaluation research: the techniques have been evaluated and implemented. Also the result of the findings discussed in the papers.

c. Solution proposal: the process point towards a unique solution that was found to a challenge. The applications and benefits of such solution are also mentioned.

d. Philosophical papers: these methods generate new ways to look at concepts and structures as problems.

e. Opinion papers: there is no known form of research for the thesis under consideration here. It simply communicates the opinions of such researchers.

f. Experience papers: such papers refer to the author's personal experience and explain how things have been done.

The classification scheme for this study considered the work in [27] suitable for use. These research types were used to examine the papers included for this study.

\subsection{Data extraction and mapping studies}

The included papers were analysed according to specific categories, based on the scheme of classification. The extraction of data from the specific documents used in the analysis was therefore possible. The classification scheme was determined by the data extraction method. New categories may be added during the process, while certain categories could be merged and some others may not be considered relevant. The data extraction process was conducted via a Spreadsheet table from Microsoft Excel. Each classification scheme category was included in the Excel tables. The publication frequency was achieved by combining the topic/contribution tables with the topic/research type tables. The frequencies from the findings on the Excel tables was the pre-requisite $\mathrm{n}$ creating the systematic map. It was important to assess which part of the research field was more prominent. In this way, the gaps in cloud middleware, stacks, and service were easily identified, allowing further work to be recommended in fields with low publications.

From the result on the spreadsheet tables, the frequencies were presented via a bubble plot, at the intersection of the categories. The map comprising the plot was an $\mathrm{x}-\mathrm{y}$ dispersion chat with bubble. The three facets used in the analysis gave rise to two quadrants. A visual map based on the contribution of the theme classifications to either the contribution/research classes was given by each quadrant. Furthermore, the bubble also provided descriptive statistics for easy comprehension. The map presented a simple overview of the analysis in the field of middleware, stacks, and services.

\section{RESULTS AND ANALYSIS}

The core motivation of the systematic mapping study on cloud computing middleware, stacks, and services was thematic analysis, classification and likely identification of the publication fora. Based on the analysis, research gaps were highlighted on the map, thus depicting which area of the field of study had shortage of publications. On the other hand, the study also indicated the areas that had more articles published. In this systematic mapping study, the primary studies were assessed via high-level classes, which were then used for producing the rates of occurrence of articles and also the map creation.

\subsection{Topic and contribution facet}

The classification scheme topics reflected the different aspects of cloud middleware and related resources. Hence the topics considered were stacks, tools, delivery network, services at all layers (XaaS), middleware and orthogonal. The tables in this study are the outcomes of the categorization of the primary studies. The primary studies were considered based on the topics used in the systematic process, hence the 
data generated are displayed on the tables. The details of this dataset are too large to be included in the paper. The numerical results in the table simply provides the quantity of papers resulting from the classification scheme and data extraction process as shown in Figure 1. This is the standard way it is represented in literature. The listing of primary studies utilized for categorizing the topics viz-a-viz the contribution type is as displayed on Table 3, while the percentage of each category is depicted in Figure 2. In Table 3, suitable topics from the papers extracted during screening and keywording are examined to determine which ones discussed metric, tool, model method and process. Hence Table 3 contains the primary studies list used for the examination of the various topics against the types of contributions. Figure 3 shows the percentage of topics in the research category. The systematic map on cloud computing middleware, stacks, tools, delivery network and services at all layers is at Figure 4. On the x-axis of the left quadrant is the contribution facet. The category dealt with the type of contribution a paper offered the research in terms of metric, method, tool, process and model. In the study, metric had $10.34 \%$ out of the 116 papers in this facet. Tool had 28.45\%, model had $27.59 \%$, method had $18.10 \%$ and process had $15.52 \%$.

Furthermore, the left quadrant indicated that $1.72 \%$ of the model contribution were each on orthogonal, middleware, delivery network and stacks. Model contributed $2.59 \%$ to services at all layers, while the contribution to tool had the largest which was $18.1 \%$. Also, Figure 2 displays the pie chart used for every topic within the contribution category. From the 116 papers within this category, $16.38 \%$ were based on stack, $51 \%$ were based on tools, $8.62 \%$ were topics on delivery network, $10.34 \%$ were topics on XaaS and $12.06 \%$ were topics on middleware.

Table 3. Primary studies for topic and contribution facets

\begin{tabular}{|c|c|c|c|c|c|}
\hline Topic $\quad$ Contribution Facet & Metric & Tool & Model & Method & Process \\
\hline Stack & $\begin{array}{c}23,24, \\
122\end{array}$ & $18,25,103,111$ & 7,26 & $\begin{array}{c}21,27,29,59 \\
110,120,126\end{array}$ & $2,45,116$ \\
\hline Tools & $\begin{array}{l}4,13,28 \\
102,105 \\
125\end{array}$ & $\begin{array}{c}31,33,65,66,67 \\
71,77,82,85,86, \\
89,91,99,100 \\
113,117\end{array}$ & $\begin{array}{c}34,37,38,39,40,60 \\
61,62,63,75,76,79 \\
80,81,82,84,104 \\
112,115,124\end{array}$ & $\begin{array}{l}32,47,56,57 \\
64,74,93\end{array}$ & $\begin{array}{c}6,10,16 \\
72,73,90 \\
113,119 \\
123\end{array}$ \\
\hline Delivery Networks & 15 & 22,48 & 14,50 & 42,43 & $51,83,127$ \\
\hline $\begin{array}{l}\text { Services at all layers (XaaS) } \\
\text { Middleware }\end{array}$ & 1,55 & $\begin{array}{c}19,20,69, \\
5,12,17,78,88, \\
92,94,107\end{array}$ & $\begin{array}{l}70,118,108 \\
9,11,49,68\end{array}$ & $\begin{array}{c}87,109 \\
30,96\end{array}$ & 98,106 \\
\hline Percentage & $10.34 \%$ & $28.45 \%$ & $27.59 \%$ & $18.10 \%$ & $15.52 \%$ \\
\hline
\end{tabular}

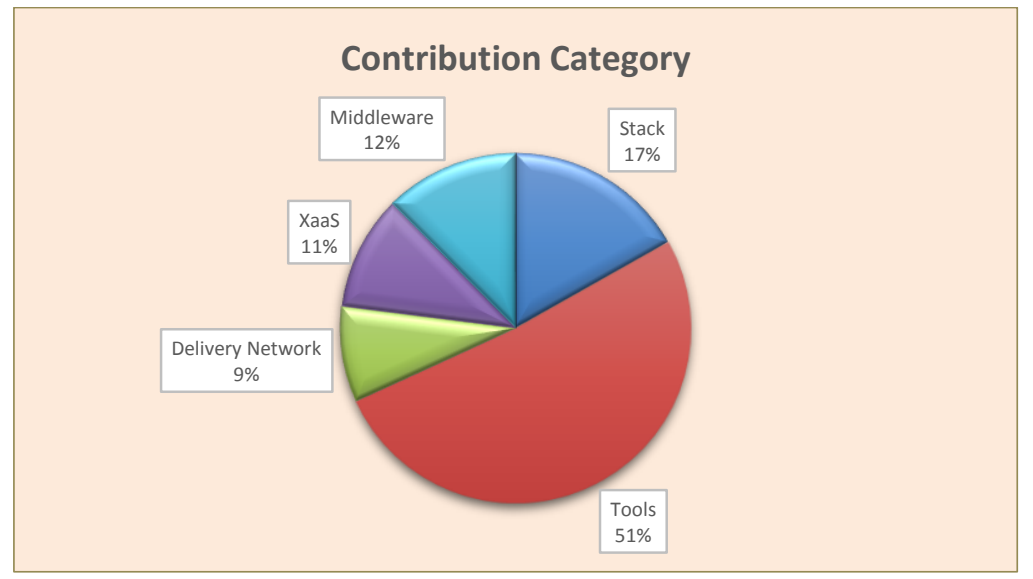

Figure 2. Percentage of topics in the contribution category

\subsection{Topic and research facet}

The listing of main studies utilized for categorization of the topics with respect to the research type is displayed on Table 4 . Table 4 contains the primary studies data used for the examination of the various topics against the types of research. In Table 4, suitable topics from the papers extracted during screening and keywording are examined to determine which ones discussed evaluation, validation, solution, philosophical, experience and opinion research types. The x-axis of the right quadrant of Figure 4 contains the result of the type of research undertaken for this study. The $\mathrm{x}$-axis of the right quadrant of Figure 4 is the research type 
category. Evaluation research had $28.35 \%$ out of 127 articles reviewed. Solution research had $54.33 \%$, philosophical had $5.15 \%$ and experience had $11.88 \%$. No publications were present on validation and opinion research in field of study under review. Evaluation research had $28.35 \%$ out of the 127 publications included in this study. The breakdown of this $28.35 \%$ indicated that $0.79 \%$ dealt with orthogonal, $6.3 \%$ of the evaluation research was on tool and 3.94\% was on stacks. From the 127 papers within this group, $15.75 \%$ were based on stack, $44.09 \%$ were on tools, $7.87 \%$ were based on delivery network, $9.45 \%$ were topics on XaaS and $22.83 \%$ were topics on middleware.

Table 4. Primary studies for topic and research facets

\begin{tabular}{|c|c|c|c|c|c|c|}
\hline Topic & Evaluation & Validation & Solution & Philosophical & Experience & Opinion \\
\hline Stack & $\begin{array}{c}23,24,103, \\
111,122\end{array}$ & & $\begin{array}{c}7,8,18,2526,27,2945, \\
59,110,120,126\end{array}$ & 21 & 2,116 & \\
\hline Tools & $\begin{array}{c}4,13,28,31 \\
33,65,66,67 \\
71,85,86,89 \\
91,99,100,102 \\
105,125\end{array}$ & & $\begin{array}{c}6,10,16,34,37,38,39,40 \\
47,56,57,60,61,62,63 \\
64,72,73,74,75,76,77,79 \\
80,81,82,84,104,112 \\
115,117,119,123,124\end{array}$ & 32,93 , & 90,113 & \\
\hline Delivery Networks & 15,48 & & $14,22,42,43,50$ & & $51,83,127$ & \\
\hline $\begin{array}{l}\text { Services at all layers } \\
\text { (XAAS) }\end{array}$ & 1,55 & & $\begin{array}{c}19,20,69,70,87,108,109 \\
118\end{array}$ & & 98,106 & \\
\hline Middleware & $\begin{array}{c}3,5,12,17,52 \\
53,97,101,121\end{array}$ & & $\begin{array}{c}9,11,49,54,68,78,88,92 \\
94,107\end{array}$ & $\begin{array}{c}30,58,114 \\
96\end{array}$ & $\begin{array}{l}35,36,41 \\
44,46,95\end{array}$ & \\
\hline Percentage & $28.35 \%$ & $0.00 \%$ & $54.33 \%$ & $5.51 \%$ & $18.81 \%$ & $0.00 \%$ \\
\hline
\end{tabular}

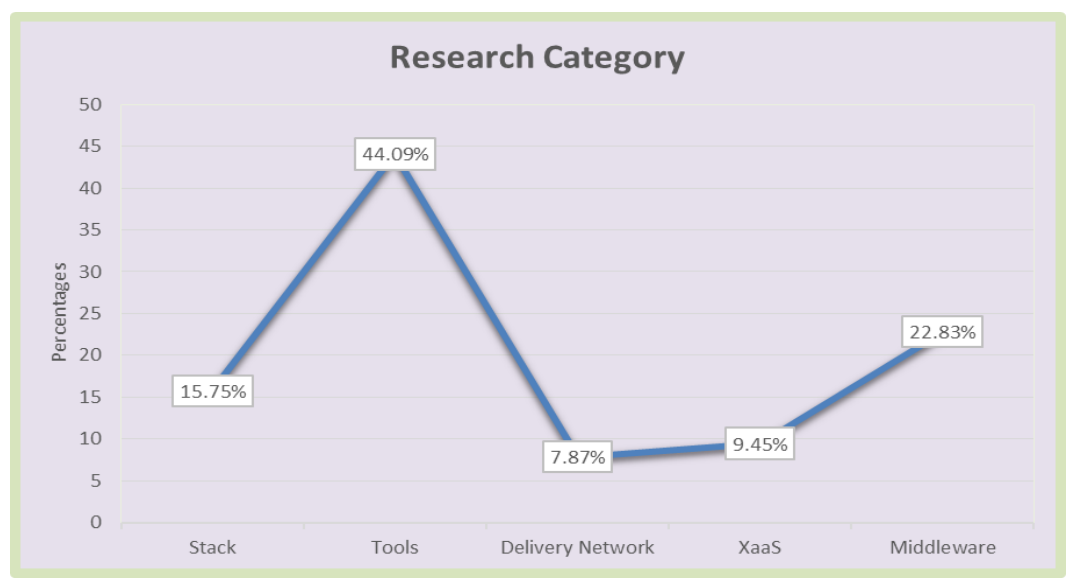

Figure 3. Percentage of topics in the research category

\subsection{Major findings}

The systematic map on middleware, stacks, tools, delivery network and services at all layers on the cloud can be visualized in Figure 4. Two x-y scatter charts that contains bubble plots at the intersection of the topic were provided in the left quadrant, at the position where the topic and contribution facets traverse. The right quadrant is the map depicting the connection of the topic and research type facet also using a two $x-y$ scatter plot with bubbles. The map made it easier to distinguish which classification had more importance. From Figure 4:

a. It is obvious that there were more papers published in the metric area as it concerns tools. In fact, it is possible to identify clearly that more publications were created on tool, as it relates to the contribution facet. Tool, model, method and process had $18.10 \%, 13.79 \%, 6.03 \%$ and $8.62 \%$ respectively.

b. Evaluation and solution research had the largest number of articles in terms of tools with $14.17 \%$ and $26.77 \%$ respectively. In terms of experience research, middleware had the highest number of papers with $4.72 \%$. Also middleware had more articles in relation to philosophical research with $2.36 \%$. There were no publications dealing with validation and opinion research based on the author's investigations. Furthermore, in terms of studies carried out, there were no papers on delivery networks and XaaS.

c. A most striking aspect of the map is that solution research has the highest frequencies of publications in relation to all aspects of the topics. This type of result shown on the systematic map should easily generate interest of researchers. The visual delivery of a systematic map has helped to summarize the 
publication gaps providing results that can be used for further studies. This is a novel work and it is hoped that more papers will be published in this field to improve on what has been done in this research paper.

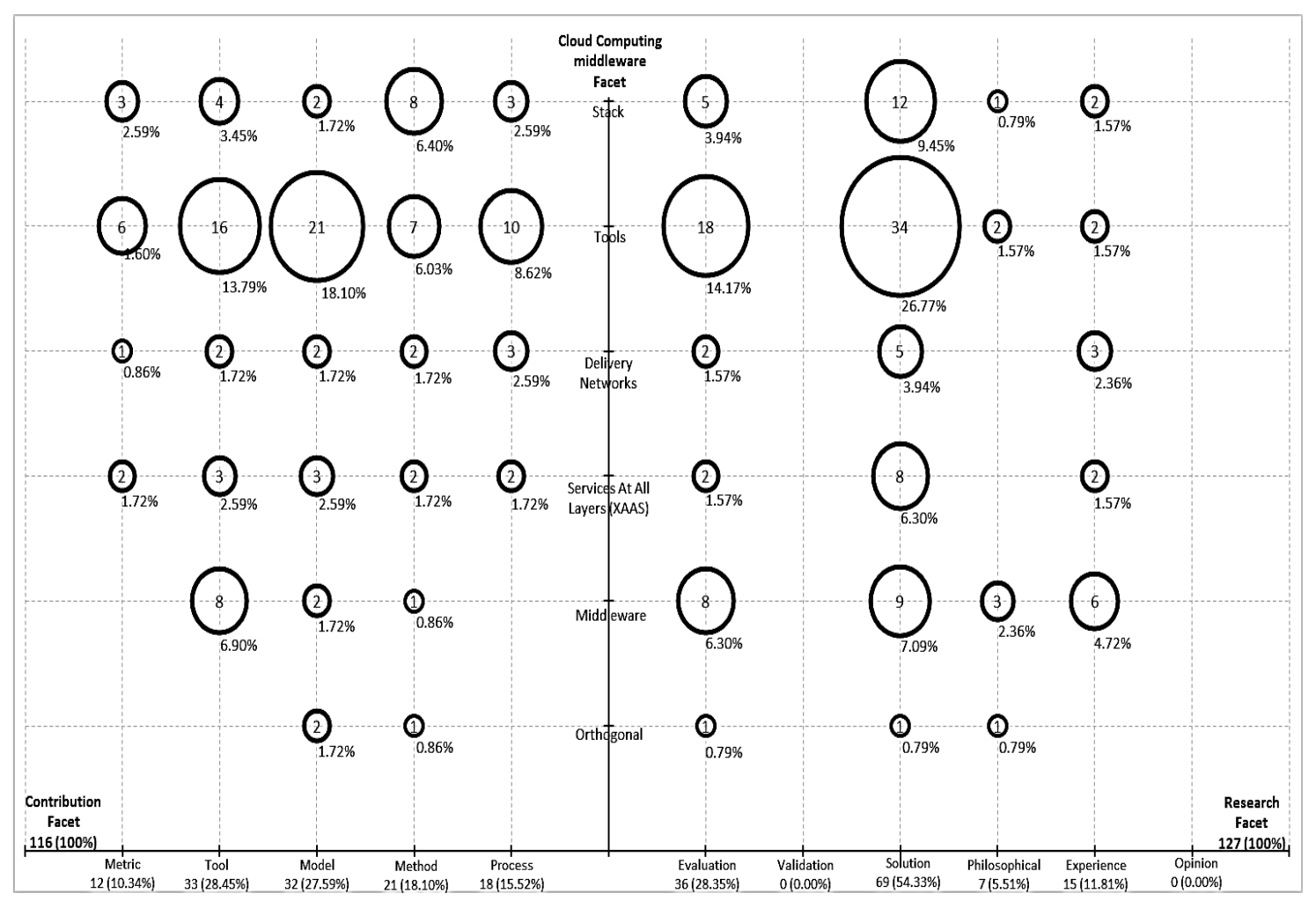

Figure 4. Systematic map of cloud computing middleware and services

The significance is that research experts at all levels could utilize this outcome for additional study. This study focused on six topics namely: stacks, tools, delivery network, services at all layers, middleware and orthogonal in relation to cloud computing middleware. In addition, six classes of study were considered either regarding tool, model, method, metric and process or in terms of evaluation, validation, solution, philosophical and opinion research. For future research these fields, among other things, are recommended. In this analysis, the essential lessons learnt is that the spectrum of research in any field is inexhaustibility. From the literature, the comparison with similar work is trivial because there has not been similar publications related to the title of this paper, hence making the contributions in this paper unique. It is expected that more upcoming researchers in the field of cloud computing will undertake more in-depth studies as a follow up to the paper.

\section{CONCLUSION}

Cloud computing is evolving very fast and so are the various challenges regarding the services being offered. Cloud users and providers alike are benefitting from cloud activities. This has inspired research efforts both within the industry and research institutions alike. The use of cloud services will require appropriate resources by prospective clients. Cloud providers must also strive to perform at an optimum level. This has led to lots of studies on cloud middleware and services. Several high impact studies were presented in this research. Notwithstanding, a shortage of publications in several aspects still exists. With the analysis carried out in the study, areas that need more work through the systematic map were highlighted. Contribution to the body of knowledge was realized by finding deficiencies in different aspects of the research. The research gaps established are useful for further studies, hence this forms the recommendation for this paper. This study can be strengthened by in-depth analysis of the topics extracted in this research when further studies are conducted. In summary, this study would enable researchers to identify important gaps in cloud middleware and services at all layers that were not addressed in past studies thus increasing research opportunities. 


\section{ACKNOWLEDGEMENTS}

We appreciate Covenant University Nigeria, through the Centre for Research, Innovation and Discovery (CUCRID) for the support granted to this work.

\section{REFERENCES}

[1] R. Buyya, J. Broberg, and A. M. Goscinski, "Cloud computing principles and paradigms," John Wiley and Son, pp. 4-10, 2011.

[2] I. Odun-Ayo, I. Eweoya, W. T.-Abasia, and S. Bogleb, “A Systematic Mapping Study of Edge Computing and Internet of Things with the Cloud," International Journal of Engineering Research and Technology, vol. 12, no. 10, pp. 1824-1836, 2019.

[3] I. Odun-Ayo, T.-A. William, J. Yahaya, and M. Odusami, "A Systematic Mapping Study of Performance Analysis and Modelling of Cloud Systems and Applications," International Journal of Electrical and Computer Engineering (IJECE), vol. 10, no. 1, pp. 101-118, 2020, doi: 10.11591/ijece.v11i2.pp1839-1848.

[4] I. Odun-Ayo, S. Misra, N. A. I. Omoregbe, E. Onibere, Y. Bulamana, and R. Damasevicius, "Cloud-based security driven human resource management system," Frontiers in Artificial Intelligence and Applications, vol. 295, pp. 96 106, 2017. doi: 10.3233/978-1-61499-773-3-96.

[5] R. Buyya, R. Ranjan and R. N. Calheiros, "Modeling and simulation of scalable Cloud computing environments and the CloudSim toolkit: Challenges and opportunities," 2009 International Conference on High Performance Computing \& Simulation, 2009, pp. 1-11, doi: 10.1109/HPCSIM.2009.5192685.

[6] A. Ranabahu, and E. M. Maximilien, "A best practice model for cloud middleware systems," IBM Almaden Research Center, San Jose, 2010.

[7] R. Buyya, S. Pandey, and C. Vecchiola, "Cloud bus toolkit for market-oriented cloud computing," in IEEE International Conference on Cloud Computing, The University of Melbourne, Australia, 2010, pp. 24-44, doi: 10.1007/978-3-642-10665-1_4.

[8] A. T. Kearney, "Clearing the fog from cloud computing," Forester, 2012.

[9] P. Subharthi, "Software-defined Application Delivery Network," All Theses and Dissertations, p. 1331, 2014, [Online]. Availabe: http://openscholarship.wustl.edu/etd/1331.

[10] K. Petersen, R. Feldt, S. Mujtaba, and M. Mattsson, "Systematic Mapping Studies in Software Engineering," EASE'08 Proceedings of the 12th international conference on Evaluation and Assessment in Software Engineering, 2008, pp. 68-77, doi: 10.5555/2227115.2227123.

[11] V. Santos, E. F. de Souza, N. L. Vijaykumar, and K. Felizardo, "Analyzing the Use of Concept Maps in Computer Science: A Systematic Mapping Study," Informatics in Education, vol. 16, no. 2, pp. 257-288, 2017. doi: 10.15388/infedu.2017.13.

[12] B. Kitchenham, O. P. Brereton, D. Budgen, M. Turner, J. Bailey, and S. Linkman, "Systematic literature reviews in software engineering-a systematic literature review," Information and Software Technology, vol. 51, no. 1, pp. 715, 2009, doi: 10.1016/j.infsof.2008.09.009.

[13] K. Petersen, S. Vakkalanka, and L. Kuzniarz, "Guidelines for conducting systematic mapping studies in software engineering: An update," Information and Software Technology, vol. 64, pp. 1-18, 2015, doi: 10.1016/j.infsof.2015.03.007.

[14] J. V. Brocke, A. Simons, K. Riemer, B. Niehaves, R. Plattfaut, and A. Cleven, "Standing on the Shoulders of Giants: Challenges and Recommendations of Literature Search in Information Systems Research," Communications of the Association for Information Systems, vol. 37, no. 1, p. 9, 2015, doi: 10.17705/1CAIS.03709.

[15] I. Odun-Ayo, R. G. Worlu, and V. Samuel, "A Systematic Mapping Study of Designs and Employment Models for Cloud: Private, Public, Hybrid, Federated and Aggregated," International Journal of Advances in Computer Science and Cloud Computing, vol. 6, no. 1, pp. 39-47, 2018.

[16] I. Odun-Ayo, I. Eweoya, W. T.-Abasi, and S. Bogle, "Networking for Cloud and Data Centre - A Systematic Mapping Study," International Journal of Engineering Research and Technology, vol. 12, no. 12, pp. 2559-2573, 2019.

[17] A. K. Mahamad, S. Saon, H. Hashim, M. A. Ahmadon, and S. Yamaguchi, "Cloud-based people counter," Bulletin of Electrical Engineering and Informatics, vol. 9, no. 1, pp. 284-291, 2020, doi: 10.11591/eei.v9i1.1849.

[18] S. Reshma and C. Chetanaprakash, "Advancement in infotainment system in automotive sector with vehicular cloud network and current state of art," International Journal of Electrical and Computer Engineering (IJECE), vol. 10, no. 2, pp. 2077-2087, 2020. doi: 10.11591/ijece.v10i2.pp2077-2087.

[19] G. Kumar and C. P. Shantala. "An extensive research survey on data integrity and deduplication towards privacy in cloud storage," International Journal of Electrical and Computer Engineering (IJECE), vol. 10, no. 2, pp. 20112022, 2020, doi: 10.11591/ijece.v10i2.pp2011-2022.

[20] A. Farahzadi, P. Shams, J. Rezazadah, and R. Farahbakhsh, "A survey of Middleware Technologies for Cloud of Things," Digital Communications and Networks, vol. 4, no. 3, pp. 176-188, 2018, doi: 10.1016/j.dcan.2017.04.005.

[21] P. T. Endol, M. Rodrigues, G. E. Goncalves, J. Kelner, D. Sadok, and C. Curescu, "High availability in clouds: systematic review and research challenges," Journal of Cloud Computing: Advances, Systems and Applications, vol. 5, no. 1, pp. 1-15, 2016, doi 10.1186/s13677-016-0066-8.

[22] E. F. Coutinho, F. R. C. Sousa, P. A. L. Rego, D. G. Gomes, and J. Souza, "Elasticity in cloud computing: a survey," annals of telecommunications - annales des télécommunications, vol. 70, no. 7-8, pp. 289-309, 2015, doi: 10.1007/s12243-014-0450-7. 
[23] M. N. Birje, P. Challagidad, R. H. Goudar, and M. T. Tapale, "Cloud computing review: concepts, technology, challenges and security," International Journal of Cloud Computing, vol. 6, no. 1, pp.32-57, 2017, doi: 10.1504/IJCC.2017.083905.

[24] M. Chiregi and N. J. Navimipour, "Cloud computing and trust evaluation: A systematic literature review of the state-of-the-art mechanisms," Journal of Electrical Systems and Information Technology, vol. 5, no. 1, pp. 608-622, 2018, doi: 10.1016/j.jesit.2017.09.001.

[25] H. J. Muhasin, R. Atan, M. A. Jabar, and S. Abdullah, "The Factors Affecting on Managing Sensitive Data in Cloud Computing," Indonesian Journal of Electrical Engineering and Computer Science, vol. 11, no. 3, pp. 11681175, 2018, doi: 10.11591/ijeecs.v11.i3.pp1168-1175.

[26] B. Kitchenham and S. Charter, "Guidelines for performing systematic literature review in software engineering," Technical report, Ver. 2.3 EBSE Technical Report. EBSE Version, vol. 2 Ed, pp. 101-117, 2007.

[27] R. Wieringa, N. A. M. Maiden, N. R. Mead, and C. Rolland, "Requirement engineering paper classification and evaluation criteria. A proposal and a discussion," Requirement Engineering, vol. 11, no. 1, pp. 102-107, 2006, doi: 10.1007/s00766-005-0021-6.

\section{BIOGRAPHIES OF AUTHORS}
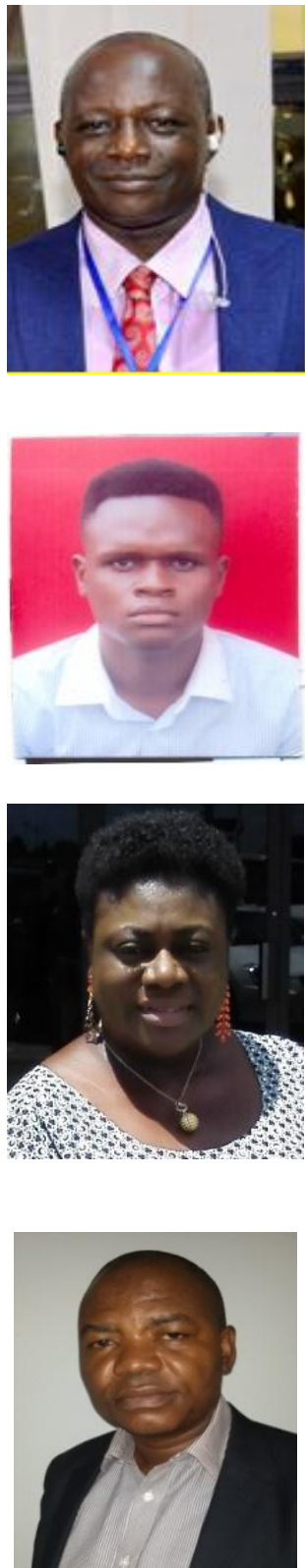

Isaac A. Odun-Ayo was born in Ilesha, Nigeria in 1962. He received the B.S, M.S and Ph.D degrees in Computer Science from the University of Benin, Benin City, Nigeria. Between 2010 and 2013 he was a faculty and Director Information and Communication Technology at the National Defence College, Abuja, Nigeria. He joined the faculty of Covenant University, Ota, Nigeria as a Senior Lecturer in October 2016. He is the author of one book and more than 40 journal and conference articles in Cloud Computing. His research interest include cloud computing, human resource management, e-governance and software engineering. Dr. OdunAyo is a recipient of the National Productivity Order of Merit Award, Nigeria for his contribution to computing. He is a member of the Nigeria Computer Society (NCS), Computer Professionals of Nigeria (CPN), International Association of Engineers (IAENG), Institute of Electrical and Electronics Engineers (IEEE) and Member Information Science Institute (ISI).

Toro-Abasi Williams received a BSc. degree in computer science from Afe Babalola University, Ado-Ekiti, Ekiti State, Nigeria in 2016. He received a MSc. Degree from the Department of Computer and Information Sciences, Covenant University, Nigeria in 2020. He takes tutorials for several courses at the postgraduate level. He has a passion for academics and research in computer science. Williams has some cloud computing publications. His research interests include cloud computing, mobile computing, artificial intelligence, machine learning, and software engineering.

Marion Adebiyi received a BSc. degree in computer science from University of Ilorin, Kwara State, Nigeria in 2000. Her MSc. and Ph. D degree also in computer science, bioinformatics Option from Covenant University, Ota, Nigeria in 2008 and 2014 respectively. She is a Senior lecturer in Computer and Information Sciences Department of Covenant University, and on deployment to Landmark University, Omu-Aran, Kwara-State, Nigeria in 2018 and currently a PDF researcher at Durban University of Technology, South Africa. She authors two books, over ten chapter in book and more than 40 articles, and 25 conferences and workshops. Dr. Adebiyi heads the H3Africa projects coordinating Division, and the Entomology and Data Management Division of Covenant University Bioinformatics Research (CUBRe) group and her research interests include bioinformatics, high throughput data analytics, genomics, proteomics, transcriptomics, homology modelling and Organism's inter-pathway analysis.

Olatunji Okesola is a Professor of Cybersecurity at the First Technical University, Ibadan Nigeria. He is a Certified Information Security Manager (CISM) and a Certified Information Systems (IS) Auditor (CISA) with a Ph.D in Computer Sciences. He is a member of IS Audit and Control Association (ISACA), Computer Professionals of Nigeria (CPN), and a fellow of Nigerian Computer Society (NCS). Okesola is a scholar, an Information Security expert and a seasoned banker. Until November 2016, he was the Group Head, for Information Systems Control and Revenue Assurance at Keystone Bank (Nig.) Ltd, Lagos. An alumni of University of South Africa, his research interests include Cyber security, biometrics, and Software engineering. He has several publications in scholarly journals and conference proceedings both local and international. 